\title{
A Fully Quantum Asymptotic Equipartition Property
}

\author{
Marco Tomamichel, ${ }^{1, *}$ Roger Colbeck, ${ }^{1,2, \text {, }}$ and Renato Renner ${ }^{1, \text { 田 }}$ \\ ${ }^{1}$ Institute for Theoretical Physics, ETH Zurich, 8093 Zurich, Switzerland. \\ ${ }^{2}$ Institute of Theoretical Computer Science, ETH Zurich, 8092 Zurich, Switzerland.
}

(Dated: May 12, 2009)

\begin{abstract}
The classical asymptotic equipartition property is the statement that, in the limit of a large number of identical repetitions of a random experiment, the output sequence is virtually certain to come from the typical set, each member of which is almost equally likely. In this paper, we prove a fully quantum generalization of this property, where both the output of the experiment and side information are quantum. We give an explicit bound on the convergence, which is independent of the dimensionality of the side information. This naturally leads to a family of Rényi-like quantum conditional entropies, for which the von Neumann entropy emerges as a special case.
\end{abstract}

\section{INTRODUCTION}

In this paper, we prove a fully quantum version of the asymptotic equipartition property (AEP). While the classical AEP applies to a random experiment with classical outcomes, here we consider the generalization to experiments that require a quantum description. Our version of the AEP then refers to typical properties of outcomes of the experiment relative to some side information, i.e., additional information obtained in the process of the experiment. We call it fully quantum because the outcomes as well as the side information may be quantum systems. We note here that in classical versions of the AEP the side information is not usually described explicitly, but is already included in the specification of the distribution of the experimental outcomes (i.e., one considers the probability distribution conditioned on the side information). This is not possible in a fully quantum context, where the side information may be entangled with the outcome and a quantum analogue of conditional probability distributions cannot be defined.

We will first discuss the classical AEP and rewrite it in a form that can then be generalized to the fully quantum setting. We sketch a possible proof of the classical version and our proof of the fully quantum AEP will follow similar lines.

\section{A. Classical AEP}

The AEP (cf. Theorem 3.1.1 in [1]) is central to classical information theory because it establishes the Shannon entropy $^{1}$,

$$
H(X)=-\sum_{x \in \mathcal{X}} P(x) \log P(x)
$$

\footnotetext{
*marcoto@phys.ethz.ch

t colbeck@phys.ethz.ch

† renner@phys.ethz.ch

1 We use log to denote the binary logarithm.
}

as the relevant quantity for various problems involving independent and identically distributed (i.i.d.) random variables. It is a direct consequence of the weak law of large numbers and states that, for large enough $n$, the outcome of a random experiment given by an i.i.d. sequence $X^{n}=\left(X_{1}, X_{2}, \ldots, X_{n}\right) \in \mathcal{X}^{\times n}$ of random variables distributed according to a probability distribution $P$ on a set $\mathcal{X}$ will almost certainly be in a set of approximately $2^{n H(X)}$ typical events that each occur with a probability close to $2^{-n H(X)}$.

Consider, for example, the problem of source compression. There, one asks for the number of bits needed to store the outcome of the above random experiment. In typical information theoretic applications, we tolerate a small probability of failure. The AEP tells us that if we ignore non-typical events, we are almost certain not to have an error. We thus only need $n H(X)$ bits to store the whole sequence, i.e. $H(X)$ bits per element.

This can alternatively be formulated in terms of the entropies

$$
\begin{aligned}
H_{\infty}(X) & :=-\log \max _{x \in \mathcal{X}} P(x) \quad \text { and } \\
H_{0}(X) & :=\log |\{x \in \mathcal{X}: P(x)>0\}| .
\end{aligned}
$$

We also informally introduce smooth min- and maxentropies denoted $H_{\text {min }}^{\varepsilon}(X)$ and $H_{\text {max }}^{\varepsilon}(X)$, which will be defined precisely in Section II. The smooth min-entropy is constructed by ignoring the most probable events in $\mathcal{X}$ up to total probability $\varepsilon$ and taking $H_{\infty}$ of the remaining distribution. Similarly, the smooth max-entropy ignores the least probable events and is closely related to $H_{0}$. In terms of these entropies, the AEP is equivalent to the relations

$$
\begin{aligned}
& \lim _{\varepsilon \rightarrow 0} \lim _{n \rightarrow \infty} \frac{1}{n} H_{\min }^{\varepsilon}\left(X^{n}\right)=H(X) \quad \text { and } \\
& \lim _{\varepsilon \rightarrow 0} \lim _{n \rightarrow \infty} \frac{1}{n} H_{\max }^{\varepsilon}\left(X^{n}\right)=H(X) .
\end{aligned}
$$

These relations have been generalized to the case of conditional entropies (for a non-asymptotic version, see [2]).

Returning to the example of source compression makes clear the second of these relations. In order to store (with certainty) the outcome of a single random experiment, 
one needs $H_{0}(X)$ bits. Furthermore, if one tolerates a small probability of failure, only roughly $H_{\max }^{\varepsilon}(X)$ bits are required. On the other hand, in the case of a large sequence of i.i.d. random variables, the AEP tells us that $H(X)$ bits are needed for each element of the sequence, and hence relation (2) follows. A similar argument can be made to illustrate relation (11) using randomness extraction [3].

We now sketch a proof of the AEP (1). We use the Rényi entropies [4]

$$
H_{\alpha}(X):=\frac{1}{1-\alpha} \log \sum_{x \in \mathcal{X}} P(x)^{\alpha}, \quad \alpha \in(0,1) \cup(1, \infty),
$$

for which $H_{\infty}(\alpha \rightarrow \infty), H_{0}(\alpha \rightarrow 0)$ and the Shannon entropy $(\alpha \rightarrow 1)$ are defined as limits. Furthermore, the entropies $H_{\alpha}$ are monotonically decreasing in $\alpha$ and, as shown in [3], the Rényi entropies with $\alpha>1$ are close to the smooth min-entropy in the sense that

$$
H_{\min }^{\varepsilon}(X) \geq H_{\alpha}(X)-\frac{1}{\alpha-1} \log \frac{1}{\varepsilon}, \quad \alpha>1
$$

while those with $\alpha<1$ are close to the smooth maxentropy. Note that the error term $\frac{1}{\alpha-1} \log 1 / \varepsilon$ in (4) diverges when we try to recover the Shannon entropy. However, in the case of an i.i.d. sequence we find

$$
\frac{1}{n} H_{\min }^{\varepsilon}\left(X^{n}\right) \geq H_{\alpha}(X)-\frac{1}{n(1-\alpha)} \log \varepsilon,
$$

where we have used $H_{\alpha}\left(X^{n}\right)=n H_{\alpha}(X)$. We proceed by bounding $\lim _{\varepsilon \rightarrow 0} \lim _{n \rightarrow \infty} H_{\text {min }}^{\varepsilon}\left(X^{n}\right)$ from above and below. To get the lower bound, we choose $\alpha=1+1 / \sqrt{n}$ and take the limit $n \rightarrow \infty$ in (5). The upper bound essentially follows from $H_{\min }(X) \leq H(X)$.

\section{B. Fully Quantum AEP}

The AEP was first generalized to situations where the outcomes A of the random experiment are quantum systems, while the side information remains classical. In this case the side information does not need to be modeled explicitly but can be included in the description of the output states (see e.g. [5, 6, 7, 8]). In this paper we consider a generalization to a fully quantum AEP, involving possibly quantum mechanical side information B. ${ }^{2}$ A preliminary version of the result has appeared in [9] (see the discussion below for a comparison). Similar

2 We consider this result an extension of the AEP in the sense that it takes the role of the classical AEP in a quantum information context. Namely, if an information theoretic problem can be solved in terms of min- and max-entropies in a single-shot scenario, the asymptotic result for i.i.d. states follows via the AEP (and, thus, can be expressed in terms of von Neumann entropies). The classical AEP follows as a special case of our result. result have also been found in the context of quantum hypothesis testing (e.g. [10, 11, 12]).

Theorem 1 (AEP). Let $\mathcal{H}_{\mathrm{A}}$ and $\mathcal{H}_{\mathrm{B}}$ be finitedimensional Hilbert spaces, $\rho_{\mathrm{AB}}$ a bipartite state on $\mathcal{H}_{\mathrm{A}} \otimes$ $\mathcal{H}_{\mathrm{B}}$ and $n \in \mathbb{N}$ s.t. $\rho_{\mathrm{AB}}^{\otimes n}$ is an i.i.d. state on $\left(\mathcal{H}_{\mathrm{A}} \otimes \mathcal{H}_{\mathrm{B}}\right)^{\otimes n}$, then

$$
\begin{aligned}
& \lim _{\varepsilon \rightarrow 0} \lim _{n \rightarrow \infty} \frac{1}{n} H_{\min }^{\varepsilon}\left(\mathrm{A}^{n} \mid \mathrm{B}^{n}\right)_{\rho^{\otimes n}}=H(\mathrm{~A} \mid \mathrm{B})_{\rho} \quad \text { and } \\
& \lim _{\varepsilon \rightarrow 0} \lim _{n \rightarrow \infty} \frac{1}{n} H_{\max }^{\varepsilon}\left(\mathrm{A}^{n} \mid \mathrm{B}^{n}\right)_{\rho \otimes n}=H(\mathrm{~A} \mid \mathrm{B})_{\rho} .
\end{aligned}
$$

This relation is expressed in terms of quantum versions of the min- and max-entropies [9] and the conditional von Neumann entropy which will be defined precisely below. The reader unfamiliar with quantum entropies is also referred to [13, 14] for many of their properties and applications.

In this contribution, we prove a non-asymptotic version of Theorem 1 that gives a lower bound on $H_{\min }^{\varepsilon}\left(\mathrm{A}^{n} \mid \mathrm{B}^{n}\right)$ for finite $n$ (cf. Theorem 9). The bound for finite $n$ has the property that the deviation from the asymptotic bound (the term $\delta(\varepsilon, \eta)$ in Theorem 9) only depends on conditional min- and max-entropies evaluated for $\rho_{\mathrm{AB}}$ but is otherwise independent of the dimension of the Hilbert spaces $\mathcal{H}_{\mathrm{A}}$ and $\mathcal{H}_{\mathrm{B}}$. More precisely, our bound is independent of the Hilbert space dimension of $\mathcal{H}_{\mathrm{B}}$. This is particularly important for applications in the context of cryptography, where quantum systems may be controlled by an adversary. In this case, it is often difficult or impossible to bound their dimension, whereas the conditional entropies can nevertheless be estimated.

It is possible to obtain a statement similar to Theorem 1 using typical subspaces. However, proofs of this type inevitably lead to bounds involving the dimensions of both Hilbert spaces $\mathcal{H}_{\mathrm{A}}$ and $\mathcal{H}_{\mathrm{B}}{ }^{3}$ and hence to a qualitatively weaker result than the one established here (in particular, no reasonable bound could be obtained for high-dimensional Hilbert spaces). We note that our proof technique is different from the one used in [9], where the result also explicitly depends on the dimension of the Hilbert space $\mathcal{H}_{\mathrm{A}}$.

Our proof is based on quantities that can be seen as a quantum generalization of Rényi entropies (Section II). A central ingredient is a family of inequalities that generalize (4) to the quantum domain (Section III). Together with a quantitative bound on the difference between the

3 To our knowledge typical subspaces cannot be defined in a fully quantum setting. Instead, it is necessary to first bound the conditional entropies in terms of (unconditional) entropies of the joint system AB and of system B separately. The typical subspace arguments can then be applied individually to get an asymptotic limit of $H(\mathrm{AB})-H(\mathrm{~B})=H(\mathrm{~A} \mid \mathrm{B})$. Bounds on the convergence derived from this argument will depend on the convergence of the individual terms and thus in general on the Hilbert space dimensions of $\mathcal{H}_{\mathrm{AB}}$ and $\mathcal{H}_{\mathrm{B}}$ (see [3]). 
generalized Rényi entropies and the von Neumann entropy (Section IV]), this leads to the main claim (Theorem 9 in Section V].

The technical tools used for the derivation of our results (in particular Lemma 14 in Appendix A) may be of independent use - for example, they allow for a simple proof of the strong sub-additivity of the von Neumann entropy (cf. Lemma [5] and [12]).

\section{QUANTUM RÉNYI ENTROPIES}

In this section, we define the various entropies used and explore some of their properties. Proofs of the lemmas can be found in Appendix B. Given a finite-dimensional Hilbert space $\mathcal{H}$, we use $\mathcal{P}(\mathcal{H})$ to denote the set of positive semi-definite operators on $\mathcal{H}$. The set of normalized quantum states $\mathcal{S}(\mathcal{H}):=\{\rho \in \mathcal{P}(\mathcal{H}): \operatorname{tr} \rho=1\}$ and the set of sub-normalized states $\mathcal{S}_{\leq}(\mathcal{H}):=\{\rho \in \mathcal{P}(\mathcal{H})$ : $\operatorname{tr} \rho \leq 1\}$ can now be defined. Indices are used to denote multi-partite Hilbert spaces, e.g. $\mathcal{H}_{\mathrm{AB}}=\mathcal{H}_{\mathrm{A}} \otimes \mathcal{H}_{\mathrm{B}}$. Let $\rho_{\mathrm{AB}} \in \mathcal{S}\left(\mathcal{H}_{\mathrm{AB}}\right)$ be a bipartite state and $\sigma_{\mathrm{B}} \in \mathcal{S}\left(\mathcal{H}_{\mathrm{B}}\right)$, then

$H(\mathrm{~A} \mid \mathrm{B})_{\rho \mid \sigma}:=\lim _{\xi \rightarrow 0} \operatorname{tr}\left(\rho_{\mathrm{AB}}\left(\mathbb{1}_{\mathrm{A}} \otimes \log \left(\sigma_{\mathrm{B}}+\xi \mathbb{1}_{\mathrm{B}}\right)-\log \rho_{\mathrm{AB}}\right)\right)$

where $\mathbb{1}_{\mathrm{A}}$ and $\mathbb{1}_{\mathrm{B}}$ are the identity operators on $\mathcal{H}_{\mathrm{A}}$ and $\mathcal{H}_{\mathrm{B}}$, respectively. The conditional von Neumann entropy can then be recovered by

$$
H(\mathrm{~A} \mid \mathrm{B})_{\rho}:=\max _{\sigma_{\mathrm{B}} \in \mathcal{S}\left(\mathcal{H}_{\mathrm{B}}\right)} H(\mathrm{~A} \mid \mathrm{B})_{\rho \mid \sigma}=H(\mathrm{~A} \mid \mathrm{B})_{\rho \mid \rho}
$$

where $\rho_{\mathrm{B}}=\operatorname{tr}_{\mathrm{A}}\left(\rho_{\mathrm{AB}}\right)$ is obtained by taking the partial trace on $A$ of $\rho_{\mathrm{AB}}$. We use indices to denote the different marginal states of multi-partite systems and often do not mention explicitly when a partial trace needs to be taken, since this information is contained implicitly in the notation of the entropies. ${ }^{4}$ We define the min-entropy:

Definition 1. Let $\rho_{\mathrm{AB}} \in \mathcal{S}_{\leq}\left(\mathcal{H}_{\mathrm{AB}}\right)$ and $\sigma_{\mathrm{B}} \in \mathcal{S}\left(\mathcal{H}_{\mathrm{B}}\right)$, then the min-entropy of $\mathrm{A}$ conditioned on $\mathrm{B}$ of the state $\rho_{\mathrm{AB}}$ relative to $\sigma_{\mathrm{B}}$ is defined as

$$
H_{\min }(\mathrm{A} \mid \mathrm{B})_{\rho \mid \sigma}:=\sup \left\{\lambda \in \mathbb{R}: 2^{-\lambda} \mathbb{1}_{\mathrm{A}} \otimes \sigma_{\mathrm{B}} \geq \rho_{\mathrm{AB}}\right\}
$$

Furthermore, we define

$$
H_{\min }(\mathrm{A} \mid \mathrm{B})_{\rho}:=\max _{\sigma_{\mathrm{B}} \in \mathcal{S}\left(\mathcal{H}_{\mathrm{B}}\right)} H_{\min }(\mathrm{A} \mid \mathrm{B})_{\rho \mid \sigma}
$$

Clearly, $H_{\min }(\mathrm{A} \mid \mathrm{B})_{\rho \mid \sigma}$ is finite if and only if $\operatorname{supp}\left\{\sigma_{\mathrm{B}}\right\} \supseteq \operatorname{supp}\left\{\rho_{\mathrm{B}}\right\}$ and $-\infty$ otherwise. The max-

\footnotetext{
4 For example, given a state $\rho_{\mathrm{ABC}}$, the entropy $H(\mathrm{~A} \mid \mathrm{B})_{\rho}$ is meant to be taken with the marginal states $\rho_{\mathrm{AB}}=\operatorname{tr}_{\mathrm{C}}\left(\rho_{\mathrm{ABC}}\right)$ and $\rho_{\mathrm{B}}=$ $\operatorname{tr}_{\mathrm{AC}}\left(\rho_{\mathrm{ABC}}\right)$.
}

entropy is its dual with regards to a purification $\rho_{\mathrm{ABC}}$ of $\rho_{\mathrm{AB}}$ on an auxiliary Hilbert space $\mathcal{H}_{\mathrm{C}}:{ }^{5}$

Definition 2. Let $\rho_{\mathrm{ABC}} \in \mathcal{S}_{\leq}\left(\mathcal{H}_{\mathrm{ABC}}\right)$ be pure, then the max-entropy of $\mathrm{A}$ conditioned on $\mathrm{B}$ of the state $\rho_{\mathrm{AB}}$ is defined as

$$
H_{\max }(\mathrm{A} \mid \mathrm{B})_{\rho}:=-H_{\min }(\mathrm{A} \mid \mathrm{C})_{\rho} .
$$

The quantum entropies can be ordered as follows:

Lemma 2. Let $\rho_{\mathrm{AB}} \in \mathcal{S}\left(\mathcal{H}_{\mathrm{AB}}\right)$, then

$$
H_{\min }(\mathrm{A} \mid \mathrm{B})_{\rho} \leq H(\mathrm{~A} \mid \mathrm{B})_{\rho} \leq H_{\max }(\mathrm{A} \mid \mathrm{B})_{\rho} .
$$

In order to define smooth versions, we consider the set of states close to $\rho$ in the following sense. For $\varepsilon>0$, we define an $\varepsilon$-ball of states around $\rho \in \mathcal{S}(\mathcal{H})$ as

$$
\mathcal{B}^{\varepsilon}(\rho):=\left\{\tilde{\rho} \in \mathcal{S}_{\leq}(\mathcal{H}): C(\rho, \tilde{\rho}) \leq \varepsilon\right\},
$$

where $C(\rho, \tilde{\rho}):=\sqrt{1-F^{2}(\rho, \tilde{\rho})}$ as proposed in [15] is a distance measure (on normalized states) based on the fidelity $F(\rho, \tilde{\rho}):=\operatorname{tr}|\sqrt{\rho} \sqrt{\tilde{\rho}}|$. We use this choice of measure because it is invariant under purifications and is directly related to the trace distance for pure states. ${ }^{6}$ Smoothed versions of the min-entropy are then defined:

$$
\begin{aligned}
H_{\min }^{\varepsilon}(\mathrm{A} \mid \mathrm{B})_{\rho \mid \sigma} & :=\max _{\tilde{\rho}_{\mathrm{AB}} \in \mathcal{B}^{\varepsilon}\left(\rho_{\mathrm{AB}}\right)} H_{\min }(\mathrm{A} \mid \mathrm{B})_{\tilde{\rho} \mid \sigma}, \\
H_{\min }^{\varepsilon}(\mathrm{A} \mid \mathrm{B})_{\rho} & :=\max _{\tilde{\rho}_{\mathrm{AB}} \in \mathcal{B}^{\varepsilon}\left(\rho_{\mathrm{AB}}\right)} H_{\min }(\mathrm{A} \mid \mathrm{B})_{\tilde{\rho}} .
\end{aligned}
$$

Similarly, we define

$$
H_{\max }^{\varepsilon}(\mathrm{A} \mid \mathrm{B})_{\rho}:=\min _{\tilde{\rho}_{\mathrm{AB}} \in \mathcal{B}^{\varepsilon}\left(\rho_{\mathrm{AB}}\right)} H_{\max }(\mathrm{A} \mid \mathrm{B})_{\tilde{\rho}} .
$$

The smoothed entropies maintain the duality relation

$$
H_{\max }^{\varepsilon}(\mathrm{A} \mid \mathrm{B})_{\rho}=-H_{\min }^{\varepsilon}(\mathrm{A} \mid \mathrm{C})_{\rho} .
$$

Both entropies are independent of the Hilbert spaces used to represent the density operators locally; namely, given $\rho_{\mathrm{AB}} \in \mathcal{S}\left(\mathcal{H}_{\mathrm{AB}}\right), \tau_{\mathrm{CD}} \in \mathcal{S}\left(\mathcal{H}_{\mathrm{CD}}\right)$ and two isometries $U$ and $V$ s.t. $\tau_{\mathrm{CD}}=(U \otimes V) \rho_{\mathrm{AB}}\left(U^{\dagger} \otimes V^{\dagger}\right)$, we have

$H_{\min }^{\varepsilon}(\mathrm{A} \mid \mathrm{B})_{\rho}=H_{\min }^{\varepsilon}(\mathrm{C} \mid \mathrm{D})_{\tau}, H_{\max }^{\varepsilon}(\mathrm{A} \mid \mathrm{B})_{\rho}=H_{\max }^{\varepsilon}(\mathrm{C} \mid \mathrm{D})_{\tau}$.

Moreover, let $\sigma_{\mathrm{B}} \in \mathcal{S}\left(\mathcal{H}_{\mathrm{B}}\right)$ and $\omega_{\mathrm{D}}:=V \sigma_{\mathrm{B}} V^{\dagger}$, then

$$
H_{\min }^{\varepsilon}(\mathrm{A} \mid \mathrm{B})_{\rho \mid \sigma}=H_{\min }^{\varepsilon}(\mathrm{C} \mid \mathrm{D})_{\tau \mid \omega} .
$$

For a more in-depth treatment of smooth conditional entropies and their basic properties, we refer to [16].

Next, we introduce a family of Rényi-like conditional entropies:

\footnotetext{
5 Note that this quantity is different from the $H_{\max }$ used in earlier work (e.g. [9]). However, the definition used here (and introduced in [14]) is chosen because it satisfies the duality relation (10). When $\mathcal{H}_{\mathrm{B}} \cong \mathbb{C}$, we recover the classical Rényi entropy $H_{1 / 2}$. Note that the smooth versions of $H_{1 / 2}$ (cf. eq. (13)) and $H_{0}$ are equivalent up to additive terms logarithmic in the smoothing parameter [3], which disappear in the asymptotic statements.

${ }^{6}$ In fact, $C(\rho, \tilde{\rho})$ corresponds to the minimal trace distance between purifications of $\rho$ and $\tilde{\rho}$ if $\operatorname{tr}(\rho)=\operatorname{tr}(\tilde{\rho})=1$.
} 
Definition 3. Let $\rho_{\mathrm{AB}} \in \mathcal{S}\left(\mathcal{H}_{\mathrm{AB}}\right), \sigma_{\mathrm{B}} \in \mathcal{S}\left(\mathcal{H}_{\mathrm{B}}\right)$ and $\alpha \in$ $(0,1) \cup(1, \infty)$, then the $\alpha$-entropy of A conditioned on B of the state $\rho_{\mathrm{AB}}$ relative to $\sigma_{\mathrm{B}}$ is given by

$$
H_{\alpha}(\mathrm{A} \mid \mathrm{B})_{\rho \mid \sigma}:=\frac{1}{1-\alpha} \log \operatorname{tr}\left(\rho_{\mathrm{AB}}^{\alpha}\left(\mathbb{1}_{\mathrm{A}} \otimes \sigma_{\mathrm{B}}\right)^{1-\alpha}\right),
$$

when $\sigma_{\mathrm{B}}$ is invertible and $\lim _{\xi \rightarrow 0} H_{\alpha}(\mathrm{A} \mid \mathrm{B})_{\rho \mid \sigma+\xi \mathbb{1}}$ otherwise.

Note that for $\alpha>1$ the limit is finite if and only if $\operatorname{supp}\left\{\sigma_{\mathrm{B}}\right\} \supseteq \operatorname{supp}\left\{\rho_{\mathrm{B}}\right\}$ and $-\infty$ otherwise. A similar quantity appears in quantum hypothesis testing [11, 17] and as a quantum relative Rényi entropy in [12, 18, 19]. If $\mathcal{H}_{\mathrm{B}} \cong \mathbb{C}$ is trivial, we recover the classical Rényi entropies (3). The entropies $H_{0}(\alpha \rightarrow 0)$ and $H_{\infty}(\alpha \rightarrow \infty)$ can be defined as limits. Moreover, the von Neumann entropy is recovered by continuous extension to $\alpha=1$ :

$$
H_{1}(\mathrm{~A} \mid \mathrm{B})_{\rho \mid \sigma}:=\lim _{\alpha \rightarrow 1} H_{\alpha}(\mathrm{A} \mid \mathrm{B})_{\rho \mid \sigma}=H(\mathrm{~A} \mid \mathrm{B})_{\rho \mid \sigma} .
$$

Unlike their classical counterparts, the quantum conditional min- and max-entropies cannot be recovered as special cases of $\alpha$-entropies. However, it can be shown [14] that, for any $\sigma_{\mathrm{B}} \in \mathcal{S}\left(\mathcal{H}_{\mathrm{B}}\right)$,

$H_{\max }(\mathrm{A} \mid \mathrm{B})_{\rho}=\max _{\tau_{\mathrm{B}} \in \mathcal{S}\left(\mathcal{H}_{\mathrm{B}}\right)} \log F^{2}\left(\rho_{\mathrm{AB}}, \mathbb{1}_{\mathrm{A}} \otimes \tau_{\mathrm{B}}\right) \geq H_{1 / 2}(\mathrm{~A} \mid \mathrm{B})_{\rho \mid \sigma}$.

Furthermore, using the eigenvalue decompositions $\rho_{\mathrm{AB}}=$ $\sum_{i} \nu_{i}|i\rangle\langle i|$ and $\mathbb{1}_{\mathrm{A}} \otimes \sigma_{\mathrm{B}}=\sum_{j} \mu_{j}|\bar{j}\rangle\langle\bar{j}|$, we have

$$
H_{\infty}(\mathrm{A} \mid \mathrm{B})_{\rho \mid \sigma}=\lim _{\xi \rightarrow 0}-\log \max _{\substack{i, j \\\langle i \mid \bar{j}\rangle \neq 0}} \frac{\nu_{i}}{\mu_{j}+\xi} \leq H_{\min }(\mathrm{A} \mid \mathrm{B})_{\rho \mid \sigma} .
$$

Nevertheless, the $\alpha$-entropies share some of the useful properties of their classical counterparts:

Lemma 3. Let $\rho_{\mathrm{AB}} \in \mathcal{S}\left(\mathcal{H}_{\mathrm{AB}}\right)$ and $\sigma_{\mathrm{B}} \in \mathcal{S}\left(\mathcal{H}_{\mathrm{B}}\right)$, then the entropies $H_{\alpha}(\mathrm{A} \mid \mathrm{B})_{\rho \mid \sigma}$ are monotonically decreasing in $\alpha$.

Furthermore, the entropies are additive, e.g. evaluation for an i.i.d. state $\rho_{\mathrm{AB}}^{\otimes n}$ relative to another i.i.d. state $\sigma_{\mathrm{B}}^{\otimes n}$ results in

$$
H_{\alpha}\left(\mathrm{A}^{n} \mid \mathrm{B}^{n}\right)_{\rho^{\otimes n} \mid \sigma^{\otimes n}}=n H_{\alpha}(\mathrm{A} \mid \mathrm{B})_{\rho \mid \sigma} .
$$

The $\alpha$-entropies are independent of the Hilbert spaces used to represent the density operators locally:

Lemma 4. Let $\rho_{\mathrm{AB}} \in \mathcal{S}\left(\mathcal{H}_{\mathrm{AB}}\right), \sigma_{\mathrm{B}} \in \mathcal{S}\left(\mathcal{H}_{\mathrm{B}}\right), \tau_{\mathrm{CD}} \in$ $\mathcal{S}\left(\mathcal{H}_{\mathrm{CD}}\right), \omega_{\mathrm{D}} \in \mathcal{S}\left(\mathcal{H}_{\mathrm{D}}\right)$ and $U, V$ isometries s.t. $\tau_{\mathrm{CD}}=$ $(U \otimes V) \rho_{\mathrm{AB}}\left(U^{\dagger} \otimes V^{\dagger}\right)$ and $\omega_{\mathrm{D}}=V \sigma_{\mathrm{B}} V^{\dagger}$. Then,

$$
H_{\alpha}(\mathrm{A} \mid \mathrm{B})_{\rho \mid \sigma}=H_{\alpha}(\mathrm{C} \mid \mathrm{D})_{\tau \mid \omega} .
$$

Conditional entropies are measures of the uncertainty about $A$ given $B$, hence we expect them to satisfy a data processing inequality, i.e. local processing by a tracepreserving completely positive map (TP-CPM) on system B cannot decrease the conditional entropy. The $\alpha$ entropies for $\alpha \in[0,2]$ have this property.
Lemma 5. Let $\alpha \in[0,2], \rho_{\mathrm{AB}} \in \mathcal{S}\left(\mathcal{H}_{\mathrm{AB}}\right), \sigma_{\mathrm{B}} \in \mathcal{S}\left(\mathcal{H}_{\mathrm{B}}\right)$, $\tau_{\mathrm{AC}} \in \mathcal{S}\left(\mathcal{H}_{\mathrm{AC}}\right), \omega_{\mathrm{C}} \in \mathcal{S}\left(\mathcal{H}_{\mathrm{C}}\right)$ and $\mathcal{E}$ a TP-CPM s.t. $\tau_{\mathrm{AC}}=$ $\mathcal{I} \otimes \mathcal{E}\left(\rho_{\mathrm{AB}}\right)$ and $\omega_{\mathrm{C}}=\mathcal{E}\left(\sigma_{\mathrm{B}}\right)$, where $\mathcal{I}$ is the identity on $\mathcal{S}\left(\mathcal{H}_{\mathrm{A}}\right)$, then

$$
H_{\alpha}(\mathrm{A} \mid \mathrm{B})_{\rho \mid \sigma} \leq H_{\alpha}(\mathrm{A} \mid \mathrm{C})_{\tau \mid \omega}
$$

When the partial trace over a subsystem takes the role of the TP-CPM, Lemma [5 is equivalent to strong subadditivity in the case of the von Neumann entropy. We find the following duality relation for $\alpha$-entropies:

Lemma 6. Let $\rho_{\mathrm{ABC}} \in \mathcal{S}\left(\mathcal{H}_{\mathrm{ABC}}\right)$ be pure and $\alpha \in[0,2]$, then

$$
H_{\alpha}(\mathrm{A} \mid \mathrm{B})_{\rho \mid \rho}=-H_{2-\alpha}(\mathrm{A} \mid \mathrm{C})_{\rho \mid \rho} .
$$

The duality relation of the von Neumann entropy$H(\mathrm{~A} \mid \mathrm{B})_{\rho}=-H(\mathrm{~A} \mid \mathrm{C})_{\rho}$ - follows in the limit $\alpha \rightarrow 1$.

\section{LOWER BOUND ON SMOOTH MIN-ENTROPY}

Our main tool for proving the fully quantum AEP is a family of inequalities that relate the smooth conditional min-entropy $H_{\min }^{\varepsilon}(\mathrm{A} \mid \mathrm{B})$ to $H_{\alpha}(\mathrm{A} \mid \mathrm{B})$ for $\alpha \in(1,2]$. The result is a quantum generalization of (4).

Theorem 7. Let $\rho_{\mathrm{AB}} \in \mathcal{S}\left(\mathcal{H}_{\mathrm{AB}}\right), \sigma_{\mathrm{B}} \in \mathcal{S}\left(\mathcal{H}_{\mathrm{B}}\right), \varepsilon>0$ and $\alpha \in(1,2]$, then the following inequality holds:

$$
H_{\min }^{\varepsilon}(\mathrm{A} \mid \mathrm{B})_{\rho \mid \sigma} \geq H_{\alpha}(\mathrm{A} \mid \mathrm{B})_{\rho \mid \sigma}-\frac{1}{\alpha-1} \log \frac{2}{\varepsilon^{2}} .
$$

Proof. We consider two cases: (1) The $\alpha$ entropy diverges and the inequality holds trivially. (2) We have $\operatorname{supp}\left\{\rho_{\mathrm{B}}\right\} \subseteq \operatorname{supp}\left\{\sigma_{\mathrm{B}}\right\}$. In this case, we can find an isometry $\mathcal{H}_{\mathrm{B}}^{\prime} \rightarrow \mathcal{H}_{\mathrm{B}}$ that maps a $\sigma_{\mathrm{B}}^{\prime}$ to $\sigma_{\mathrm{B}}$ and $\rho_{\mathrm{AB}}^{\prime}$ to $\rho_{\mathrm{AB}}$ s.t. $\sigma_{\mathrm{B}}^{\prime}$ has full support. The min- and $\alpha$-entropies are invariant under this isometry due to (15) and Lemma 4, thus, we henceforth assume that $\sigma_{\mathrm{B}}$ is invertible in this proof.

We use Appendix C to get a first bound on $H_{\min }^{\varepsilon}(\mathrm{A} \mid \mathrm{B})$; in particular, let $\lambda$ be chosen s.t. Lemma 15 holds for $\varepsilon$ (cf. Remark 16). Next, we introduce the operator $X:=\rho_{\mathrm{AB}}-\lambda \mathbb{1}_{\mathrm{A}} \otimes \sigma_{\mathrm{B}}$ with eigenbasis $\left\{\left|\psi_{i}\right\rangle\right\}_{i \in S}$. The set $S^{+} \subseteq S$ contains the indices $i$ corresponding to positive eigenvalues of $X$. Hence, $P^{+}:=\sum_{i \in S^{+}}\left|\psi_{i}\right\rangle\left\langle\psi_{i}\right|$ is the projector on the positive eigenspace of $X$ and $P^{+} X P^{+}=\Delta$ as defined in Lemma [15. Furthermore, let $r_{i}:=\left\langle\psi_{i}\left|\rho_{\mathrm{AB}}\right| \psi_{i}\right\rangle \geq 0$ and $s_{i}:=\left\langle\psi_{i}\left|\mathbb{1}_{\mathrm{A}} \otimes \sigma_{\mathrm{B}}\right| \psi_{i}\right\rangle>0$. It follows that

$$
\forall_{i \in S^{+}}: r_{i}-\lambda s_{i} \geq 0 \quad \text { and } \quad \frac{r_{i}}{\lambda s_{i}} \geq 1
$$


For any $\alpha \in(1,2]$, we bound $\varepsilon$ in Lemma 15 as follows:

$$
\begin{aligned}
\frac{\varepsilon^{2}}{2} & =\operatorname{tr}(\Delta)=\sum_{i \in S^{+}} r_{i}-\lambda s_{i} \leq \sum_{i \in S^{+}} r_{i} \\
& \leq \sum_{i \in S^{+}} r_{i}\left(\frac{r_{i}}{\lambda s_{i}}\right)^{\alpha-1} \\
& \leq \lambda^{1-\alpha} \sum_{i \in S} r_{i}^{\alpha} s_{i}^{1-\alpha} .
\end{aligned}
$$

Next, we apply Lemma 14 to the functional $S_{g_{\alpha}}$, where $g_{\alpha}: t \mapsto t^{\alpha}$ is operator convex for $\alpha \in(1,2]$ (cf. Section V.2 of [20]). We use the TP-CPM $A \mapsto$ $\sum_{i \in S}\left|\psi_{i}\right\rangle\left\langle\psi_{i}|A| \psi_{i}\right\rangle\left\langle\psi_{i}\right|$ to obtain

$$
S_{g_{\alpha}}\left(\rho_{\mathrm{AB}}, \mathbb{1}_{\mathrm{A}} \otimes \sigma_{\mathrm{B}}\right)=\operatorname{tr}\left(\rho_{\mathrm{AB}}^{\alpha}\left(\mathbb{1}_{\mathrm{A}} \otimes \sigma_{\mathrm{B}}\right)^{1-\alpha}\right) \geq \sum_{i \in S} r_{i}^{\alpha} s_{i}^{1-\alpha} .
$$

Substituting this into (23), we find

$$
\lambda^{\alpha-1} \leq \frac{2}{\varepsilon^{2}} \operatorname{tr}\left(\rho_{\mathrm{AB}}^{\alpha}\left(\mathbb{1}_{\mathrm{A}} \otimes \sigma_{\mathrm{B}}\right)^{1-\alpha}\right) .
$$

Finally, taking the logarithm on both sides, dividing by $1-\alpha<0$ and applying Lemma 15 results in (22).

\section{LOWER BOUND ON $\alpha$-ENTROPIES}

We will use Theorem 7 to get a lower bound on the min-entropy in terms of $\alpha$-entropies, hence, it remains to find a lower bound on the $\alpha$-entropies in terms of the von Neumann entropy. In turn, the bound on the convergence will depend on the smoothing parameter $\varepsilon$ and a contribution $\Upsilon(\mathrm{A} \mid \mathrm{B})$ that describes how fast the $\alpha$-entropies converge to the von Neumann entropy.

Definition 4. Let $\rho_{\mathrm{AB}} \in \mathcal{S}\left(\mathcal{H}_{\mathrm{AB}}\right)$ and $\sigma_{\mathrm{B}} \in \mathcal{S}\left(\mathcal{H}_{\mathrm{B}}\right)$, then we define the $\alpha$-entropy convergence parameter

$$
\Upsilon(\mathrm{A} \mid \mathrm{B})_{\rho \mid \sigma}:=2^{-\frac{1}{2} H_{3 / 2}(\mathrm{~A} \mid \mathrm{B})_{\rho \mid \sigma}}+2^{\frac{1}{2} H_{1 / 2}(\mathrm{~A} \mid \mathrm{B})_{\rho \mid \sigma}}+1 .
$$

When $\sigma_{\mathrm{B}}=\rho_{\mathrm{B}}$, one can use (17) and its dual relation $H_{3 / 2}(\mathrm{~A} \mid \mathrm{B})_{\rho \mid \rho} \geq H_{\min }(\mathrm{A} \mid \mathrm{B})_{\rho}$ (cf. (10) and Lemma 6) to write

$$
\Upsilon(\mathrm{A} \mid \mathrm{B})_{\rho \mid \rho} \leq \sqrt{2^{-H_{\min }(\mathrm{A} \mid \mathrm{B})_{\rho}}}+\sqrt{2^{H_{\max }(\mathrm{A} \mid \mathrm{B})_{\rho}}}+1
$$

We can now state a bound on the $\alpha$-entropies for $\alpha$ close to 1 as follows:

Lemma 8. Let $\rho_{\mathrm{AB}} \in \mathcal{S}\left(\mathcal{H}_{\mathrm{AB}}\right), \sigma_{\mathrm{B}} \in \mathcal{S}\left(\mathcal{H}_{\mathrm{B}}\right), \quad \eta=$ $\Upsilon(A \mid B)_{\rho \mid \sigma}$ and $1<\alpha<1+\frac{\log 3}{4 \log \eta}$, then the following inequality holds:

$$
H_{\alpha}(\mathrm{A} \mid \mathrm{B})_{\rho \mid \sigma} \geq H(\mathrm{~A} \mid \mathrm{B})_{\rho \mid \sigma}-4(\alpha-1)(\log \eta)^{2} .
$$

Proof. We assume that $\sigma_{\mathrm{B}}$ is invertible in this proof. The general result then follows by the arguments outlined at the beginning of the proof of Theorem 7 .
Let $\{|i\rangle\}_{i}$ be an orthonormal basis of $\mathcal{H}_{\mathrm{AB}}$ and $\mathcal{H}_{\mathrm{AB}}^{\prime} \cong$ $\mathcal{H}_{\mathrm{AB}}$ a copy of $\mathcal{H}_{\mathrm{AB}}$. The state $|\gamma\rangle:=\sum_{i}|i\rangle \otimes|i\rangle$ is the (unnormalized) fully entangled state on $\mathcal{H}_{\mathrm{AB}} \otimes \mathcal{H}_{\mathrm{AB}}^{\prime}$. We introduce a purification $|\phi\rangle:=\left(\sqrt{\rho_{\mathrm{AB}}} \otimes \mathbb{1}_{\mathrm{AB}}\right)|\gamma\rangle$ of $\rho_{\mathrm{AB}}$. To simplify notation, we use $\beta:=\alpha-1$ as well as $X:=\rho_{\mathrm{AB}} \otimes\left(\mathbb{1} \otimes \sigma_{\mathrm{B}}^{-1}\right)^{T}$.

Let us first approximate $H_{\alpha}(\mathrm{A} \mid \mathrm{B})$ for small $\beta>0$ :

$H_{\alpha}(\mathrm{A} \mid \mathrm{B})_{\rho \mid \sigma}=-\frac{1}{\beta} \log \left\langle\phi\left|X^{\beta}\right| \phi\right\rangle \geq \frac{1}{\beta \ln 2}\left(1-\left\langle\phi\left|X^{\beta}\right| \phi\right\rangle\right)$,

where we used $\ln x \leq x-1$ for all $x>0$. We now expand the exponential $t^{\beta}$ for each eigenvalue $t>0$ of $X$ as follows: $t^{\beta}=1+\beta \ln t+r_{\beta}(t)$, where $r_{\beta}(t):=$ $t^{\beta}-\beta \ln t-1$. This leads to

$$
\begin{aligned}
H_{\alpha}(\mathrm{A} \mid \mathrm{B})_{\rho \mid \sigma} & \geq \frac{1}{\beta \ln 2}\left(-\beta\langle\phi|\ln X| \phi\rangle-\left\langle\phi\left|r_{\beta}(X)\right| \phi\right\rangle\right) \\
& =H(\mathrm{~A} \mid \mathrm{B})_{\rho \mid \sigma}-\frac{1}{\beta \ln 2}\left\langle\phi\left|r_{\beta}(X)\right| \phi\right\rangle
\end{aligned}
$$

To simplify this further, we note that

$$
r_{\beta}(t) \leq 2(\cosh (\beta \ln t)-1)=: s_{\beta}(t) .
$$

It is easy to verify that $s_{\beta}$ is monotonically increasing for $t \geq 1$ and concave in $t$ for $\beta \leq 1 / 2$ and $t \in[3, \infty)$. Furthermore, we have $s_{\beta}(t)=s_{\beta}\left(\frac{1}{t}\right)$ and $s_{\beta}\left(t^{2}\right)=s_{2 \beta}(t)$. We use this to write ${ }^{7}$

$$
\begin{aligned}
s_{\beta}(t) & \leq s_{\beta}\left(t+\frac{1}{t}+2\right)=s_{2 \beta}\left(\sqrt{t}+\frac{1}{\sqrt{t}}\right) \\
& \leq s_{2 \beta}\left(\sqrt{t}+\frac{1}{\sqrt{t}}+1\right) .
\end{aligned}
$$

Next, we apply (27) to the matrix element in (26) and use the fact that the operator $\sqrt{X}+1 / \sqrt{X}+\mathbb{1}$ has its eigenvalues in $[3, \infty)$ and $2 \beta<\frac{\log 3}{2 \log \eta} \leq \frac{1}{2}$ together with Lemma 11 in Appendix A:

$$
\left\langle\phi\left|r_{\beta}(X)\right| \phi\right\rangle \leq\left\langle\phi\left|s_{2 \beta}\left(\sqrt{X}+\frac{1}{\sqrt{X}}+\mathbb{1}\right)\right| \phi\right\rangle \leq s_{2 \beta}(\eta),
$$

where we substituted $\eta=\langle\phi|\sqrt{X}+1 / \sqrt{X}+\mathbb{1}| \phi\rangle$. Taylor's theorem and an expansion around $\beta=0$ gives an upper bound on $s_{\beta}(t): s_{\beta}(t) \leq \beta^{2}(\ln t)^{2} \cosh (\beta \ln t)$. Hence,

$$
\begin{aligned}
\frac{1}{\beta \ln 2} s_{2 \beta}(\eta) & \leq 4 \beta(\log \eta)^{2} \ln 2 \cosh (2 \beta \ln \eta) \\
& <4 \beta(\log \eta)^{2}
\end{aligned}
$$

where we simplified the expression (for convenience of exposition) using $\ln 2 \cosh (\ln 3 / 2)<1$. The lemma now follows after we substitute (29) and (28) into (26).

\footnotetext{
7 Adaptions of this step lead to different bounds. Here, we are interested in a bound that can be expressed in terms of $H_{1 / 2}$ and $H_{3 / 2}$.
} 


\section{QUANTUM AEP}

One could use Theorem 7 , together with the arguments given for the classical case in Section IA, to prove (6) directly. In many applications, it is useful to have an explicit lower bound on $H_{\min }^{\varepsilon}(\mathrm{A} \mid \mathrm{B})$. We derive such a bound, from which the asymptotic version (6) is a corollary.

Theorem 9. Let $\rho_{\mathrm{AB}} \in \mathcal{S}\left(\mathcal{H}_{\mathrm{AB}}\right), \varepsilon>0, \eta=\Upsilon(A \mid B)_{\rho \mid \rho}$ and $n \in \mathbb{N}$ s.t. $\rho_{\mathrm{AB}}^{\otimes n}$ is an i.i.d. state on $\mathcal{H}_{\mathrm{AB}}^{\otimes n}$, then

$$
\frac{1}{n} H_{\min }^{\varepsilon}\left(\mathrm{A}^{n} \mid \mathrm{B}^{n}\right)_{\rho}^{\otimes n} \geq H(\mathrm{~A} \mid \mathrm{B})_{\rho}-\frac{\delta(\varepsilon, \eta)}{\sqrt{n}},
$$

where, for $n \geq \frac{8}{5} \log \frac{2}{\varepsilon^{2}}$, the error term is given by

$$
\delta(\varepsilon, \eta):=4 \log \eta \sqrt{\log \frac{2}{\varepsilon^{2}}} .
$$

Proof. By definition, we have

$$
\frac{1}{n} H_{\min }^{\varepsilon}\left(\mathrm{A}^{n} \mid \mathrm{B}^{n}\right)_{\rho^{\otimes n}} \geq \frac{1}{n} H_{\min }^{\varepsilon}\left(\mathrm{A}^{n} \mid \mathrm{B}^{n}\right)_{\rho} \otimes n \mid \rho^{\otimes n} .
$$

We use Theorem 7, the additivity property (18) of the $\alpha$-entropy and Lemma 8 to get a bound on the rhs. Let $\alpha:=1+\frac{1}{2 \mu \sqrt{n}}$ for a parameter $\mu$ (to be optimized over), then

$$
\begin{aligned}
\text { rhs. } & \geq \frac{1}{n} H_{\alpha}\left(\mathrm{A}^{n} \mid \mathrm{B}^{n}\right)_{\rho^{\otimes n} \mid \rho \otimes n}-\frac{1}{n(\alpha-1)} \log \frac{2}{\varepsilon^{2}} \\
& =H_{\alpha}(\mathrm{A} \mid \mathrm{B})_{\rho \mid \rho}-\frac{2 \mu}{\sqrt{n}} \log \frac{2}{\varepsilon^{2}} \\
& \geq H(\mathrm{~A} \mid \mathrm{B})_{\rho \mid \rho}-\frac{2}{\sqrt{n}}\left(\mu \log \frac{2}{\varepsilon^{2}}+\frac{1}{\mu}(\log \eta)^{2}\right) .
\end{aligned}
$$

We want to choose $\mu$ such that it minimizes the expression $\mu \log \frac{2}{\varepsilon^{2}}+\mu^{-1}(\log \eta)^{2}$. However, the requirement $\alpha<1+\frac{\log 3}{4 \log \eta}$ in Lemma 8 restricts the choice of $\mu$ for any fixed $n$, hence, the error term $\delta(\varepsilon, \eta)$ is in general also a function of $n$. Nonetheless, for large enough $n$ the optimum, $\mu_{*}$, can be reached ${ }^{8}$ and we get

$$
\mu_{*}=\sqrt{\frac{(\log \eta)^{2}}{\log \frac{2}{\varepsilon^{2}}}} \quad \text { for } \quad n \geq \frac{8}{5} \frac{(\log \eta)^{2}}{\mu_{*}^{2}}=\frac{8}{5} \log \frac{2}{\varepsilon^{2}} .
$$

Substitution of this expression into (31) leads to (30).

Remark 10. The proof of Theorem 9 can be generalized to $H_{\min }(\mathrm{A} \mid \mathrm{B})_{\rho \mid \sigma}$. The generalized theorem reads: For $\rho_{\mathrm{AB}} \in \mathcal{S}\left(\mathcal{H}_{\mathrm{AB}}\right)$ and $\sigma_{\mathrm{B}} \in \mathcal{S}\left(\mathcal{H}_{\mathrm{B}}\right)$, we have

$$
\frac{1}{n} H_{\min }^{\varepsilon}\left(\mathrm{A}^{n} \mid \mathrm{B}^{n}\right)_{\rho^{\otimes n} \mid \sigma^{\otimes n}} \geq H(\mathrm{~A} \mid \mathrm{B})_{\rho \mid \sigma}-\frac{\delta(\varepsilon, \eta)}{\sqrt{n}},
$$

\footnotetext{
8 To verify this, evaluate an upper bound to $\alpha=1+\left(2 \mu_{*} \sqrt{n}\right)^{-1}$ using the expression for $n$ in (32) and note that $\sqrt{5 / 2}<\log 3$.
}

where $n$ and $\delta(\varepsilon, \eta)$ as in Theorem 9 and $\eta=\Upsilon(\mathrm{A} \mid \mathrm{B})_{\rho \mid \sigma} \cdot{ }^{9}$

The generalized asymptotic equipartition property stated in Theorem 1 follows as a corollary.

Proof of Theorem 1, We first show the min-entropy relation (6). Taking the $n \rightarrow \infty$ limit in Theorem 9 gives

$$
\lim _{\varepsilon \rightarrow 0} \lim _{n \rightarrow \infty} \frac{1}{n} H_{\min }^{\varepsilon}\left(\mathrm{A}^{n} \mid \mathrm{B}^{n}\right)_{\rho \otimes n} \geq H(\mathrm{~A} \mid \mathrm{B})_{\rho} .
$$

However, since, for $\varepsilon \rightarrow 0$, the min-entropy is smaller than the Shannon entropy (cf. Lemma 2), we get the desired result. To see this, note that there exists a $\tilde{\rho}_{\mathrm{A}^{n} \mathrm{~B}^{n}} \in \mathcal{B}^{\varepsilon}\left(\rho_{\mathrm{AB}}^{\otimes n}\right)$ s.t.

$$
H_{\min }^{\varepsilon}\left(\mathrm{A}^{n} \mid \mathrm{B}^{n}\right)_{\rho^{\otimes n}}=H_{\min }\left(\mathrm{A}^{n} \mid \mathrm{B}^{n}\right)_{\tilde{\rho}} \leq H\left(\mathrm{~A}^{n} \mid \mathrm{B}^{n}\right)_{\tilde{\rho}} .
$$

We now use the continuity of the von Neumann entropy under small perturbations of the state as expressed in Fannes' inequality (cf. [22]). This ensures that, for finitedimensional Hilbert spaces, the difference between the Shannon entropies evaluated for $\rho_{\mathrm{AB}}^{\otimes n}$ and $\tilde{\rho}_{\mathrm{A}^{n} \mathrm{~B}^{n}}$ scales at most linearly in $n$ (i.e. logarithmic in the Hilbert space dimension):

$$
\lim _{n \rightarrow \infty} \frac{1}{n}\left|H\left(\mathrm{~A}^{n} \mid \mathrm{B}^{n}\right)_{\tilde{\rho}}-H\left(\mathrm{~A}^{n} \mid \mathrm{B}^{n}\right)_{\rho^{\otimes n}}\right| \leq O(\varepsilon) .
$$

Together with (33), this leads to

$$
\lim _{\varepsilon \rightarrow 0} \lim _{n \rightarrow \infty} \frac{1}{n} H_{\min }^{\varepsilon}\left(\mathrm{A}^{n} \mid \mathrm{B}^{n}\right)_{\rho}^{\otimes n} \leq H(\mathrm{~A} \mid \mathrm{B})_{\rho} .
$$

The max-entropy relation (7) follows after we substitute the duals of the smooth min-entropy (14) and the von Neumann entropy (21) into (6).

\section{APPENDIX A: TECHNICAL RESULTS}

We discuss some properties of convex and operator convex $^{10}$ functions. Let $\mathcal{H}, \mathcal{H}^{\prime}$ be Hilbert spaces and $|\phi\rangle \in \mathcal{H}$. We start with a straightforward application of Jensen's inequality:

Lemma 11. Let $f$ be a convex function on $[a, b]$ and $X$ an operator on $\mathcal{H}$ s.t. $a \mathbb{1} \leq X \leq b \mathbb{1}$. Then,

$$
\langle\phi|f(X)| \phi\rangle \geq f(\langle\phi|X| \phi\rangle)
$$

\footnotetext{
${ }^{9}$ Furthermore, note that all results of this paper can also be stated in terms of relative entropies instead of conditional entropies. For example, in the language of [21], Remark 10 reads: Let $\rho, \sigma \in \mathcal{H}$ and $\varepsilon>0$. With $n$ and $\delta$ as in Theorem 9 we have $\frac{1}{n} D_{\max }^{\varepsilon}\left(\rho^{\otimes n} \| \sigma^{\otimes n}\right) \leq S(\rho \| \sigma)+\delta(\varepsilon, \eta) / \sqrt{n}$, where the smoothing is over an $\varepsilon$-ball around $\rho$ as defined in the present work and $\eta=2^{1 / 2 S_{3 / 2}(\rho \| \sigma)}+2^{-1 / 2 S_{1 / 2}(\rho \| \sigma)}+1$.

10 A continuous function $f$ on $[0, \infty)$ is operator convex if $f\left(\frac{1}{2}(A+\right.$ $B)) \leq \frac{1}{2}(f(A)+f(B))$ for all positive semi-definite matrices $A$ and $B$.
} 
A generalization of Jensen's inequality to operator convex functions was shown in [23]:

Lemma 12 (Operator Jensen's Inequality). Let $f$ be a continuous operator convex function on $[0, \infty)$ and $\nu$ an isometry $\mathcal{H} \rightarrow \mathcal{H}^{\prime}$. Then, for all $C \geq 0$ on $\mathcal{H}^{\prime}$ it holds that

$$
\nu^{\dagger} f(C) \nu \geq f\left(\nu^{\dagger} C \nu\right)
$$

We will now discuss Lemma 14, originally proven by Petz [24], which establishes the monotonicity of certain functionals $S_{f}$ under TP-CPMs and is of independent use in quantum information theory (see e.g. [11, 12]). Let $\mathcal{H}$ be a Hilbert space with orthonormal basis $\{|i\rangle\}_{i}$, $\mathcal{H}^{\prime} \cong \mathcal{H}$ be a copy of $\mathcal{H}$, and $|\gamma\rangle:=\sum_{i}|i\rangle \otimes|i\rangle$ be the (unnormalized) fully entangled state on $\mathcal{H} \otimes \mathcal{H}^{\prime}$. Then, for any continuous function $f:[0, \infty) \rightarrow \mathbb{R}$ with $f(0)=0$, and operators $A \geq 0, B>0$ on $\mathcal{H}$, we define

$$
S_{f}(A, B):=\left\langle\gamma\left|(\sqrt{B} \otimes \mathbb{1}) f\left(B^{-1} \otimes A^{T}\right)(\sqrt{B} \otimes \mathbb{1})\right| \gamma\right\rangle,
$$

where $(\cdot)^{T}$ denotes the transpose with respect to $\{|i\rangle\}_{i}$ and $\mathbb{1}$ is the identity operator on $\mathcal{H}$. More generally, for any $B \geq 0$, we define

$$
S_{f}(A, B)=\lim _{\xi \rightarrow 0} S_{f}(A, B+\xi \mathbb{1}) .
$$

The functional $S_{f}(A, B)$ is independent of the Hilbert space used to represent $A$ and $B$ in the following sense:

Lemma 13. Let $U: \mathcal{H} \rightarrow \overline{\mathcal{H}}$ be an isometry, then for all operators $A \geq 0, B \geq 0$ on $\mathcal{H}$ :

$$
S_{f}(A, B)=S_{f}\left(U A U^{\dagger}, U B U^{\dagger}\right) .
$$

Proof. Let $A=\sum_{i} \lambda_{i}|\underline{i}\rangle\langle\underline{i}|$ and $B=\sum_{j} \mu_{j}|\bar{j}\rangle\langle\bar{j}|$ with eigenvalues $\lambda_{i} \geq 0, \mu_{j} \geq 0$ and orthonormal bases $\{|\underline{i}\rangle\}_{i}$ and $\{|\bar{j}\rangle\}_{j}$ respectively. Now, using $\langle\gamma|X \otimes \mathbb{1}| \gamma\rangle=\operatorname{tr}(X)$ and $(X \otimes \mathbb{1})|\gamma\rangle=\left(\mathbb{1} \otimes X^{T}\right)|\gamma\rangle$ for any operator $X$, we get

$$
S_{f}(A, B)=\lim _{\xi \rightarrow 0} \sum_{i, j}\left(\mu_{j}+\xi\right) f\left(\frac{\lambda_{i}}{\mu_{j}+\xi}\right)|\langle\underline{i} \mid \bar{j}\rangle|^{2} .
$$

The isometry $U$ keeps the eigenvalues and the scalar product $\langle\underline{i} \mid \bar{j}\rangle$ invariant. Furthermore, any zero eigenvalues introduced do not contribute to the sum since they lie on a space orthogonal to the image of $U$, and $f(0)=0$.

Lemma 14. Let $f$ be operator convex on $[0, \infty)$ and let $\mathcal{E}$ be a TP-CPM, then for all operators $A \geq 0, B \geq 0$ on $\mathcal{H}$ :

$$
S_{f}(A, B) \geq S_{f}(\mathcal{E}(A), \mathcal{E}(B)) .
$$

Proof. Every TP-CPM can be expressed as an isometry followed by a partial trace (cf. [8], Section 8.2). We have already established in Lemma 13 that $S_{f}$ is invariant under isometries. To show monotonicity under partial trace, we let $\mathcal{H}=\mathcal{H}_{1} \otimes \mathcal{H}_{2}$ with local bases $\left\{|i\rangle_{1}\right\}_{i}$ and $\left\{|i\rangle_{2}\right\}_{i}$, respectively. We introduce $\mathcal{H}_{1}^{\prime} \cong \mathcal{H}_{1}$, the (unnormalized) fully entangled state $|\gamma\rangle=\sum_{i, j}|i\rangle_{1} \otimes|j\rangle_{2} \otimes|i\rangle_{1} \otimes$ $|j\rangle_{2}$ and its marginal $|\gamma\rangle_{1}=\sum_{i}|i\rangle_{1} \otimes|i\rangle_{1}$. It remains to show that $S_{f}(A, B) \geq S_{f}\left(A_{1}, B_{1}\right)$, where $A_{1}=\operatorname{tr}_{2}(A)$ and $B_{1}=\operatorname{tr}_{2}(B)$. We will show this under the assumption that $B$ is invertible and the result for general $B$ will follow from the continuity (by definition) of $S_{f}$ when $\xi \rightarrow 0$.

Let us define a linear map $\nu: \mathcal{H}_{1} \otimes \mathcal{H}_{1}^{\prime} \rightarrow \mathcal{H} \otimes \mathcal{H}^{\prime}$ by

$$
\nu:=\sum_{i}\left(\sqrt{B}\left({\sqrt{B_{1}}}^{-1} \otimes|i\rangle_{2}\right)\right) \otimes \mathbb{1}_{1} \otimes|i\rangle_{2} .
$$

The map $\nu$ is an isometry, i.e. $\nu^{\dagger} \nu=\mathbb{1}_{11}$ and satisfies

$$
\nu\left(\sqrt{B_{1}} \otimes \mathbb{1}_{1}|\gamma\rangle_{1}\right)=\sqrt{B} \otimes \mathbb{1}_{12}|\gamma\rangle .
$$

Moreover, we have $\operatorname{tr}_{2}\left(A^{T}\right)=A_{1}^{T}$, since the transpose is taken in the product basis. Hence, it follows that

$$
\nu^{\dagger}\left(B^{-1} \otimes A^{T}\right) \nu=B_{1}^{-1} \otimes A_{1}^{T} .
$$

Next, we apply Lemma 12 to get

$\nu^{\dagger} f\left(B^{-1} \otimes A^{T}\right) \nu \geq f\left(\nu^{\dagger}\left(B^{-1} \otimes A^{T}\right) \nu\right)=f\left(B_{1}^{-1} \otimes A_{1}^{T}\right)$.

Finally, using (A2), we recover $S_{f}(A, B) \geq S_{f}\left(A_{1}, B_{1}\right)$ by taking the matrix element for $\left(\sqrt{B_{1}} \otimes \mathbb{1}_{1}\right)|\gamma\rangle_{1}$ on both sides of the inequality.

\section{APPENDIX B: PROOFS OF CLAIMS IN SECTION II}

For completeness, we prove various properties of the min-, max- and $\alpha$-entropies presented in Section I.

Proof of Lemma 2. It is sufficient to prove the first relation $^{11}$, since $H_{\min }(\mathrm{A} \mid \mathrm{B}) \leq H(\mathrm{~A} \mid \mathrm{B})$ implies $H_{\max }(\mathrm{A} \mid \mathrm{C}) \geq$ $H(\mathrm{~A} \mid \mathrm{C})$ by the duality relations.

$$
\begin{aligned}
H(\mathrm{~A} \mid \mathrm{B})_{\rho} & =\max _{\sigma_{\mathrm{B}} \in \mathcal{S}\left(\mathcal{H}_{\mathrm{B}}\right)} \operatorname{tr}\left(\rho_{\mathrm{AB}}\left(\log \left(\mathbb{1}_{\mathrm{A}} \otimes \sigma_{\mathrm{B}}\right)-\log \rho_{\mathrm{AB}}\right)\right) \\
& \geq \operatorname{tr}\left(\rho_{\mathrm{AB}}\left(\log \left(\lambda \mathbb{1}_{\mathrm{A}} \otimes \sigma_{\mathrm{B}}^{\prime}\right)-\log \rho_{\mathrm{AB}}\right)\right)-\log \lambda \\
& \geq H_{\min }(\mathrm{A} \mid \mathrm{B})_{\rho},
\end{aligned}
$$

where we chose $\lambda>0$ and $\sigma_{\mathrm{B}}^{\prime} \in \mathcal{S}\left(\mathcal{H}_{\mathrm{B}}\right)$ such that they optimize (8) and (9). Hence, $-\log \lambda=H_{\min }(\mathrm{A} \mid \mathrm{B})_{\rho}$. Furthermore, it follows from (8) that $\lambda \mathbb{1}_{\mathrm{A}} \otimes \sigma_{\mathrm{B}}^{\prime} \geq \rho_{\mathrm{AB}}$. Then, using the operator monotonicity ${ }^{12}$ of $t \mapsto \log t$ (cf. Chapter $\mathrm{V}$ in [20]), we find that the remaining term is positive.

${ }^{11}$ See also Lemma 10 in 21] for an alternative proof. There they define the relative entropy $D_{\max }\left(\rho_{\mathrm{B}} \| \sigma_{\mathrm{B}}\right)$ which, for $\mathcal{H}_{\mathrm{A}} \cong \mathbb{C}$ trivial, is equal to $-H_{\min }(\mathrm{A} \mid \mathrm{B})_{\rho \mid \sigma}$.

12 A function $f$ on $(0, \infty)$ is operator monotone if $A \geq B$ implies $f(A) \geq f(B)$ for any strictly positive Hermitian matrices $A$ and $B$. 
Proof of Lemma 3. We prove this statement for invertible $\sigma_{\mathrm{B}}$ and the general statement then follows by continuity. Using the (unnormalized) fully entangled state $|\gamma\rangle$ as in Appendix A, we define a purification $|\phi\rangle:=$ $\left(\sqrt{\rho_{\mathrm{AB}}} \otimes \mathbb{1}_{\mathrm{AB}}\right)|\gamma\rangle$ of $\rho_{\mathrm{AB}}$. Furthermore, we set $\beta:=\alpha-1$ and $X:=\rho_{\mathrm{AB}} \otimes\left(\mathbb{1} \otimes \sigma_{\mathrm{B}}^{-1}\right)^{T}$. It is easy to verify that, for $f: t \mapsto t \log t$,

$$
\begin{aligned}
H_{\alpha}(\mathrm{A} \mid \mathrm{B})_{\rho \mid \sigma} & =-\frac{1}{\beta} \log \left\langle\phi\left|X^{\beta}\right| \phi\right\rangle \quad \text { and } \\
\frac{\partial}{\partial \alpha} H_{\alpha}(\mathrm{A} \mid \mathrm{B})_{\rho \mid \sigma} & =\frac{1}{\beta^{2}} \log \left\langle\phi\left|X^{\beta}\right| \phi\right\rangle-\frac{1}{\beta} \frac{\left\langle\phi\left|X^{\beta} \log X\right| \phi\right\rangle}{\left\langle\phi\left|X^{\beta}\right| \phi\right\rangle} \\
& =\frac{f\left(\left\langle\phi\left|X^{\beta}\right| \phi\right\rangle\right)-\left\langle\phi\left|f\left(X^{\beta}\right)\right| \phi\right\rangle}{\beta^{2}\left\langle\phi\left|X^{\beta}\right| \phi\right\rangle} .
\end{aligned}
$$

The statement of the lemma then follows from the convexity of $f$ together with Lemma 11.

Proof of Lemma 4. The $\alpha$-entropies can be expressed in terms of the functionals of Appendix A. Given the continuous functions $g_{\alpha}: t \mapsto t^{\alpha}$ and $h: t \mapsto-t \log t$ that satisfy $g_{\alpha}(0)=0$ for $\alpha \in(0, \infty)$ and $h(0)=0$, we write

$$
H_{\alpha}(\mathrm{A} \mid \mathrm{B})_{\rho \mid \sigma}=\left\{\begin{array}{ll}
\frac{1}{1-\alpha} \log S_{g_{\alpha}}\left(\rho_{\mathrm{AB}}, \mathbb{1}_{\mathrm{A}} \otimes \sigma_{\mathrm{B}}\right) & \alpha \in(0,1) \\
S_{h}\left(\rho_{\mathrm{AB}}, \mathbb{1}_{\mathrm{A}} \otimes \sigma_{\mathrm{B}}\right) & \alpha=1 \\
-\frac{1}{\alpha-1} \log S_{g_{\alpha}}\left(\rho_{\mathrm{AB}}, \mathbb{1}_{\mathrm{A}} \otimes \sigma_{\mathrm{B}}\right) & \alpha \in(1, \infty)
\end{array} .\right.
$$

The proof is now a straightforward application of Lemma 13 with isometry $U \otimes V$ to the functionals $S_{h}$ and $S_{g_{\alpha}}$. Note that $(U \otimes V)\left(\mathbb{1}_{\mathrm{A}} \otimes \sigma_{\mathrm{B}}\right)\left(U^{\dagger} \otimes V^{\dagger}\right)=U U^{\dagger} \otimes \omega_{\mathrm{D}}$, where $U U^{\dagger}$ and $\mathbb{1}_{\mathrm{C}}$ are interchangeable in the definition of $H_{\alpha}(\mathrm{C} \mid \mathrm{D})_{\tau \mid \omega}$, since $\tau_{\mathrm{C}}$ has its support on $U U^{\dagger}$. The statements for $\alpha \rightarrow 0$ and $\alpha \rightarrow \infty$ follow by continuity.

Proof of Lemma 5. The proof is a straightforward application of Lemma 14 with TP-CPM $\mathcal{I} \otimes \mathcal{E}$ to the functionals in (B1). In the von Neumann limit $\alpha=1$, we write

$$
\begin{aligned}
H(\mathrm{~A} \mid \mathrm{B})_{\rho \mid \sigma} & =S_{h}\left(\rho_{\mathrm{AB}}, \mathbb{1}_{\mathrm{A}} \otimes \sigma_{\mathrm{B}}\right) \\
& \leq S_{h}\left(\mathcal{I} \otimes \mathcal{E}\left(\rho_{\mathrm{AB}}\right), \mathcal{I} \otimes \mathcal{E}\left(\mathbb{1}_{\mathrm{A}} \otimes \sigma_{\mathrm{B}}\right)\right) \\
& =S_{h}\left(\tau_{\mathrm{AC}}, \mathbb{1}_{\mathrm{A}} \otimes \omega_{\mathrm{C}}\right)=H(\mathrm{~A} \mid \mathrm{C})_{\tau \mid \omega},
\end{aligned}
$$

where we used that $h: t \mapsto-t \log t$ is operator concave on $[0, \infty)$ (cf. Chapter V in [20]). Similarly, we use that $g_{\alpha}: t \mapsto t^{\alpha}$ on $[0, \infty)$ is operator concave for $\alpha \in(0,1)$ and operator convex for $\alpha \in(1,2]$ (cf. Chapter V in [20]) as well as continuity at $\alpha=0$ to prove the statement for $\alpha \in[0,2]$.

Proof of Lemma 6. We write $\rho_{\mathrm{ABC}}=|\vartheta\rangle\langle\vartheta|$ and note that the marginal states $\rho_{\mathrm{AB}}$ and $\rho_{\mathrm{C}}$ satisfy $\left(\rho_{\mathrm{AB}} \otimes \mathbb{1}_{\mathrm{C}}\right)|\vartheta\rangle=$ $\left(\mathbb{1}_{\mathrm{AB}} \otimes \rho_{\mathrm{C}}\right)|\vartheta\rangle$. The same applies to $\rho_{\mathrm{B}}$ and $\rho_{\mathrm{AC}}$. Thus,

$$
\begin{aligned}
& (1-\alpha) H_{\alpha}(\mathrm{A} \mid \mathrm{B})_{\rho \mid \rho} \\
& \quad=\log \operatorname{tr}\left(\rho_{\mathrm{AB}}^{\alpha}\left(\mathbb{1}_{\mathrm{A}} \otimes \rho_{\mathrm{B}}\right)^{1-\alpha}\right) \\
& \quad=\log \left\langle\vartheta\left|\left(\rho_{\mathrm{AB}} \otimes \mathbb{1}_{\mathrm{C}}\right)^{\alpha-1}\left(\mathbb{1}_{\mathrm{A}} \otimes \rho_{\mathrm{B}} \otimes \mathbb{1}_{\mathrm{C}}\right)^{1-\alpha}\right| \vartheta\right\rangle \\
& \quad=\log \operatorname{tr}\left(\left(\mathbb{1}_{\mathrm{A}} \otimes \rho_{\mathrm{C}}\right)^{\alpha-1} \rho_{\mathrm{AC}}^{2-\alpha}\right) .
\end{aligned}
$$

The equality now follows from $\alpha-1=1-(2-\alpha)$.

\section{APPENDIX C: ESTIMATE OF $H_{\min }^{\varepsilon}(\mathrm{A} \mid \mathrm{B})_{\rho}$}

The following lemma gives an estimate of the smooth min-entropy $H_{\min }^{\varepsilon}(\mathrm{A} \mid \mathrm{B})_{\rho}$ (see also [25]):

Lemma 15. Let $\rho_{\mathrm{AB}} \in \mathcal{S}\left(\mathcal{H}_{\mathrm{AB}}\right), \sigma_{\mathrm{B}} \in \mathcal{S}\left(\mathcal{H}_{\mathrm{B}}\right)$ and $\lambda>0$, then

$$
H_{\min }^{\varepsilon}(\mathrm{A} \mid \mathrm{B})_{\rho \mid \sigma} \geq-\log \lambda, \quad \varepsilon=\sqrt{2 \operatorname{tr}(\Delta)},
$$

where $\Delta:=\left\{\rho_{\mathrm{AB}}-\lambda \mathbb{1}_{\mathrm{A}} \otimes \sigma_{\mathrm{B}}\right\}_{+}$is the positive part of the operator $\rho_{\mathrm{AB}}-\lambda \mathbb{1}_{\mathrm{A}} \otimes \sigma_{\mathrm{B}}$.

Proof. We first choose $\tilde{\rho}_{\mathrm{AB}}$, bound $H_{\min }(\mathrm{A} \mid \mathrm{B})_{\tilde{\rho} \mid \sigma}$, and then show that $\tilde{\rho}_{\mathrm{AB}} \in \mathcal{B}^{\varepsilon}\left(\rho_{\mathrm{AB}}\right)$. We use the abbreviated notation $\Lambda:=\lambda \mathbb{1}_{\mathrm{A}} \otimes \sigma_{\mathrm{B}}$ and set

$$
\tilde{\rho}_{\mathrm{AB}}:=G \rho_{\mathrm{AB}} G^{\dagger}, \quad G:=\Lambda^{\frac{1}{2}}(\Lambda+\Delta)^{-\frac{1}{2}},
$$

where the inverse is taken on the support of $\Lambda$. From the definition of $\Delta$, we have $\rho_{\mathrm{AB}} \leq \Lambda+\Delta$; hence, $\tilde{\rho}_{\mathrm{AB}} \leq \Lambda$ and $H_{\min }(\mathrm{A} \mid \mathrm{B})_{\tilde{\rho} \mid \sigma} \geq-\log \lambda$.

Let $|\psi\rangle$ be a purification of $\rho_{\mathrm{AB}}$, then $\left(G \otimes \mathbb{1}_{\mathrm{AB}}\right)|\psi\rangle$ is a purification of $\tilde{\rho}_{\mathrm{AB}}$ and, using Uhlmann's theorem, we find a bound on the fidelity:

$F\left(\rho_{\mathrm{AB}}, \tilde{\rho}_{\mathrm{AB}}\right) \geq\left|\left\langle\psi\left|G \otimes \mathbb{1}_{\mathrm{AB}}\right| \psi\right\rangle\right| \geq \Re\left\{\operatorname{tr}\left(G \rho_{\mathrm{AB}}\right)\right\}=\operatorname{tr}\left(\bar{G} \rho_{\mathrm{AB}}\right)$,

where we introduced $\bar{G}:=\frac{1}{2}\left(G+G^{\dagger}\right)$. Hence,

$$
C\left(\rho_{\mathrm{AB}}, \tilde{\rho}_{\mathrm{AB}}\right) \leq \sqrt{\left(1+\operatorname{tr}\left(\bar{G} \rho_{\mathrm{AB}}\right)\right)\left(1-\operatorname{tr}\left(\bar{G} \rho_{\mathrm{AB}}\right)\right)} .
$$

This can be simplified further after we note that $G$ is a contraction. ${ }^{13}$ To see this, we multiply $\Lambda \leq \Lambda+\Delta$ with $(\Lambda+\Delta)^{-\frac{1}{2}}$ from left and right to get

$$
G^{\dagger} G=(\Lambda+\Delta)^{-\frac{1}{2}} \Lambda(\Lambda+\Delta)^{-\frac{1}{2}} \leq \mathbb{1}_{\mathrm{AB}} .
$$

Furthermore, $\bar{G} \leq \mathbb{1}_{\mathrm{AB}}$, since $\|\bar{G}\| \leq 1$ by the triangle inequality and $\|G\|=\left\|G^{\dagger}\right\| \leq 1$. Clearly, $\operatorname{tr}\left(\bar{G} \rho_{\mathrm{AB}}\right) \leq 1$. Moreover,

$$
\begin{aligned}
1-\operatorname{tr}\left(\bar{G} \rho_{\mathrm{AB}}\right) & =\operatorname{tr}\left(\left(\mathbb{1}_{\mathrm{AB}}-\bar{G}\right) \rho_{\mathrm{AB}}\right) \\
& \leq \operatorname{tr}(\Lambda+\Delta)-\operatorname{tr}(\bar{G}(\Lambda+\Delta)) \\
& =\operatorname{tr}(\Lambda+\Delta)-\operatorname{tr}\left((\Lambda+\Delta)^{1 / 2} \Lambda^{1 / 2}\right) \\
& \leq \operatorname{tr}(\Delta),
\end{aligned}
$$

where we used $\rho_{\mathrm{AB}} \leq \Lambda+\Delta$ and $\sqrt{\Lambda+\Delta} \geq \sqrt{\Lambda}$. The latter inequality follows from the operator monotonicity of the square root function (Proposition V.1.8 in [20]). Finally, $C\left(\rho_{\mathrm{AB}}, \tilde{\rho}_{\mathrm{AB}}\right) \leq \sqrt{2 \operatorname{tr}(\Delta)}=\varepsilon$ and $\tilde{\rho}_{\mathrm{AB}} \in \mathcal{B}^{\varepsilon}\left(\rho_{\mathrm{AB}}\right)$.

13 A contraction $G$ is an operator with operator norm $\|G\| \leq 1$. 
Remark 16. For a fixed $\varepsilon \in[0,1]$ and $\operatorname{supp}\left\{\rho_{\mathrm{B}}\right\} \subseteq$ $\operatorname{supp}\left\{\sigma_{\mathrm{B}}\right\}$, we can always find a finite $\lambda$ s.t. Lemma 15 holds. To see this, note that

$$
\varepsilon(\lambda)=\sqrt{2 \operatorname{tr}\left\{\rho_{\mathrm{AB}}-\lambda \mathbb{1}_{\mathrm{A}} \otimes \sigma_{\mathrm{B}}\right\}_{+}}
$$

is continuous in $\lambda$ with $\varepsilon(0)=\sqrt{2}$ and $\varepsilon\left(2^{-H_{\min }(\mathrm{A} \mid \mathrm{B})_{\rho \mid \sigma}}\right)=0$.

\section{ACKNOWLEDGMENTS}

We thank Johan Åberg, Nilanjana Datta, Milán Mosonyi and Jürg Wullschleger for comments. We acknowledge support from the Swiss National Science Foundation (grant No. 200021-119868).
[1] T. M. Cover and J. A. Thomas, Elements of Information Theory. Wiley Series in Telecommunications, 1991.

[2] T. Holenstein and R. Renner, "On the randomness of independent experiments," 2006. [Online]. Available: http://arxiv.org/abs/cs/0608007

[3] R. Renner and S. Wolf, "Smooth Rényi entropy and applications," in Proc. ISIT, 2004.

[4] A. Rényi, "On measures of information and entropy," in Proc. 4th Berkeley Symp. on Math., Stat. and Prob., 1961, pp. 547-561.

[5] R. Jozsa and B. Schumacher, "A new proof of the quantum noiseless coding theorem," J. Mod. Opt., vol. 41, pp. 2343-2349, 1994.

[6] H. Barnum, E. Knill, and M. Nielsen, "On quantum fidelities and channel capacities," IEEE Trans. Inf. Theory, vol. 46, no. 4, pp. 1317-1329, Jul. 2000.

[7] B. Schoenmakers, J. Tjoelker, P. Tuyls, and E. Verbitskiy, "Smooth Rényi entropy of ergodic quantum information sources," in Proc. ISIT, 200\%, pp. 256-260.

[8] M. A. Nielsen and I. L. Chuang, Quantum Computation and Quantum Information. Cambridge University Press, 2000.

[9] R. Renner, "Security of quantum key distribution," Ph.D. dissertation, Swiss Federal Institute of Technology, Zurich, 2005. [Online]. Available: http://arxiv.org/abs/quant-ph/0512258

[10] F. Hiai and D. Petz, "The proper formula for relative entropy and its asymptotics in quantum probability," Commun. Math. Phys., vol. 143, pp. 99-114, 1991.

[11] T. Ogawa and H. Nagaoka, "Strong converse and Stein's lemma in quantum hypothesis testing," IEEE Trans. Inf. Theory, vol. 46, pp. 2428-2433, Nov. 2000.

[12] M. Hayashi, Quantum Information - An Introduction. Springer, 2006.

[13] R. Renner and R. König, "Universally composable privacy amplification against quantum adversaries," in Proc. TCC, 2005, pp. 407-425. [Online]. Available: http://arxiv.org/abs/quant-ph/0403133

[14] R. König, R. Renner, and C. Schaffner, "The operational meaning of min- and max-entropy," Jul. 2008. [Online]. Available: http://arxiv.org/abs/0807.1338

[15] A. Gilchrist, N. K. Langford, and M. A. Nielsen, "Distance measures to compare real and ideal quantum processes," Aug. 2004. [Online]. Available: http://arxiv.org/abs/quant-ph/0408063

[16] M. Tomamichel, R. Colbeck, and R. Renner, "On the smoothing of conditional min- and max-entropies," Unpublished Notes, 2009.

[17] K. M. R. Audenaert, J. Calsamiglia, R. Munoz-Tapia, E. Bagan, L. Masanes, A. Acin, and F. Verstraete, "Discriminating states: The quantum chernoff bound," Phys. Rev. Lett., vol. 98, no. 16, p. 160501, 2007.

[18] M. Ohya and D. Petz, Quantum Entropy and Its Use. Springer, 1993.

[19] M. Mosony and N. Datta, "Generalized relative entropies and the capacity of classical-quantum channels," 2008. [Online]. Available: http://arxiv.org/abs/0810.3478

[20] R. Bhatia, Matrix Analysis, ser. Graduate Texts in Mathematics. Springer, 1997.

[21] N. Datta, "Min- and max- relative entropies and a new entanglement monotone," Mar. 2008. [Online]. Available: http://arxiv.org/abs/0803.2770v1

[22] R. Alicki and M. Fannes, "Continuity of quantum mutual information," Dec. 2003. [Online]. Available: http://arxiv.org/abs/quant-ph/0312081v1

[23] F. Hansen and G. K. Pedersen, "Jensen's operator inequality," Bull. London Math. Soc., vol. 35, no. 5, pp. 553-564, Jul. 2003.

[24] D. Petz, "Quasi-entropies for finite quantum systems," Rep. Math. Phys., vol. 23, no. 1, pp. 57-65, Sep. 1984.

[25] N. Datta and R. Renner, "Smooth Rényi entropies and the quantum information spectrum," Jan. 2008. [Online]. Available: http://arxiv.org/abs/0801.0282 